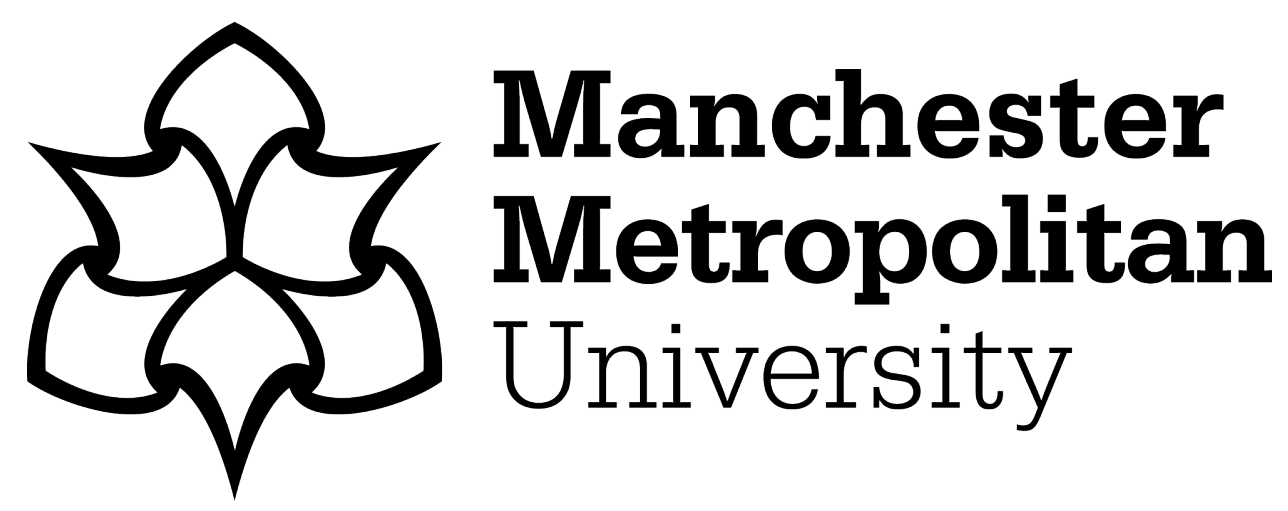

$\mathrm{Li}$, YongSheng, Chen, Jing, Wang, LiLi, Guo, Yuan, Feng, Jiling and Chen, WeiYi (2018) Experimental Verification of the Elastic Formula for the Aspirated Length of a Single Cell Considering the Size and Compressibility of Cell During Micropipette Aspiration. Annals of Biomedical Engineering, 46 (7). pp. 1026-1037. ISSN 0090-6964

Downloaded from: https://e-space.mmu.ac.uk/622468/

Version: Accepted Version

Publisher: Springer Nature

DOI: https://doi.org/10.1007/s10439-018-2023-9

Please cite the published version 


\title{
Experimental Verification of the Elastic Formula for the Aspirated Length of a Single Cell Considering the Size and Compressibility of Cell During Micropipette Aspiration
}

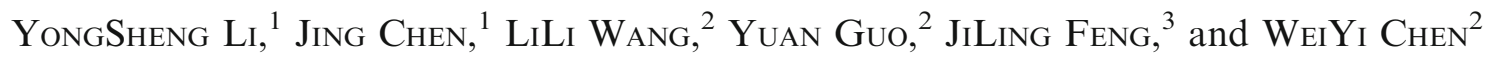 \\ ${ }^{1}$ Department of Mechanics and Engineering Science, Taiyuan University of Technology, 79 West Yingze Street, Taiyuan 030024, \\ China; ${ }^{2}$ Institute of Applied Mechanics and Biomedical Engineering, Taiyuan University of Technology, 79 West Yingze Street, \\ Taiyuan 030024, China; and ${ }^{3}$ Department of Design, Manufacture, and Engineering Management, University of Strathclyde, \\ Glasgow G1 1XQ, Scotland, UK
}

(Received 30 October 2017; accepted 3 April 2018; published online 10 April 2018)

Associate Editor Sean S. Kohles oversaw the review of this article.

\begin{abstract}
In this study, an aspiration system for elastic spheres was developed to verify the approximate elastic formula for the aspirated length of a single solid-like cell undergoing micropipette aspiration (MPA), which was obtained in our previous study by theoretical analysis and numerical simulation. Using this system, foam silicone rubber spheres with different diameters and mechanical properties were aspirated in a manner similar to the MPA of single cells. Comparisons between the approximate elastic formula and aspiration experiments of spheres indicated that the predictions of the formula agreed with the experimental results. Additionally, combined with the MPA data of rabbit chondrocytes, differences in terms of the elastic parameters derived from the halfspace model, incompressible sphere model, and compressible sphere model were explored. The results demonstrated that the parameter $\xi(\xi=R / a$, where $R$ is the radius of the cell and $a$ is the inner radius of the micropipette) and Poisson's ratio significantly influenced the determination of the elastic modulus and bulk modulus of the cell. This work developed for the first time an aspiration system of elastic spheres to study the elastic responses of the MPA of a single cell and provided new evidence supporting the use of the approximate elastic formula to determine cellular elastic parameters from the MPA data.
\end{abstract}

Keywords-Cell mechanics, Micropipette aspiration, Cell model, Mechanical properties, Experimental verification.

\section{INTRODUCTION}

Mechanical properties of cells play an important role in defining cell functionality, motility, tissue formation, ${ }^{8,35}$ stem cell differentiation, ${ }^{6}$ etc. Studies into

Address correspondence to WeiYi Chen, Institute of Applied Mechanics and Biomedical Engineering, Taiyuan University of Technology, 79 West Yingze Street, Taiyuan 030024, China. Electronic mail: 819903@163.com mechanics of single cells have been rapidly evolved during past decades with significant implications towards human health. It has been implicated in the pathogenesis of many progressive diseases, including vascular disease, kidney disease, cancer, malaria, cataracts, cardiomyopathies, etc. ${ }^{10}$ For instance, higher possibility of heart failure is due to loss of contractility of heart muscle cells. ${ }^{7}$ The stiffening of red blood cells infected with malaria, ${ }^{22,26}$ was found to be responsible for fatal incidents of this disease. When erythrocyte stiffens causing vessel occlusion, patients might suffer from coma and this may lead to death. Low rigidity of cancer cells was recently suggested as an indicator for cancer diagnosis. ${ }^{19,28}$ On the whole, cell's mechanical properties represent the physical structure of living cells and have been proved to be effective as label-free biomarkers for detecting abnormalities in diseased cells. ${ }^{18}$ Therefore, in addition to the fundamental interest, there is a practical need to measure cell mechanics quantitatively.

In the past, various techniques have been developed for a single cell's mechanical characterization such as atomic force microscopy (AFM) ${ }^{29}$ optical trapping $(\mathrm{OT}),{ }^{25}$ magnetic twisting cytometry (MTC), ${ }^{24}$ micropipette aspiration (MPA), ${ }^{13}$ microelectromechanical systems (MEMS), ${ }^{23}$ and microfluidics. ${ }^{1}$ As a traditional approach, MPA is still widely employed in the evaluation of a single cell's mechanical properties for its high precision and wide range. ${ }^{31,32,36}$ In MPA, a cell is aspirated by a small negative pressure into a small glass tube with an inner diameter smaller than the cell. Subsequently, the length of the cell projection in the pipette, the pressure applied, and the duration of 
observation are recorded. Then, the elastic and viscoelastic properties of the cell can be evaluated. Various models have been developed to extract the average mechanical properties of anchorage-dependent cells (so-called solid-like cells, e.g., endothelial cells and chondrocytes) from the MPA data. ${ }^{3,12,33,40}$ The halfspace model (HSM), in which a cell is treated as a semiinfinite body regardless of its size and compressibility, is widely used for its intuitive concept and expression in mechanics. 9,16,27,32,36,39 However, for the MPA of solid-like cells, such as those involving chondrocytes, the diameter of the cells is approximately $19.5 \mu \mathrm{m}$, whereas the inner diameter of the micropipette is approximately $14.1 \mu \mathrm{m}$, as shown in Fig. 1. Evidently, the geometric scales of cell and micropipette are at the same order of magnitude. Moreover, the effect of compressibility on the deformation behavior of cells is usually studied by varying Poisson's ratio $v$ from 0.2 to $0.5,3,5,15$ which indicates that the cells have significant compressibility.

Obviously, the HSM and the actual configuration [i.e. sphere model (SM), as shown in Fig. 2a] exhibit significant differences. Many studies have used numerical approaches to investigate the influences of size and compressibility on the deformation behavior of cells. Haider and Guilak ${ }^{11,12}$ investigated the elastic and viscoelastic responses of cells during MPA by using the boundary element method, which considers finite cell dimension, cell boundary curvature, and cellmicropipette contact but not cell compressibility. Zhou et $a l .{ }^{40}$ simulated the MPA of cells by using the standard neo-Hookean solid model, in which three relationships are derived and cell compressibility is neglected in interpreting the mechanical parameters of cells. Baaijens et $a .^{2}$ determined the mechanical properties of chondrocytes by using the finite element

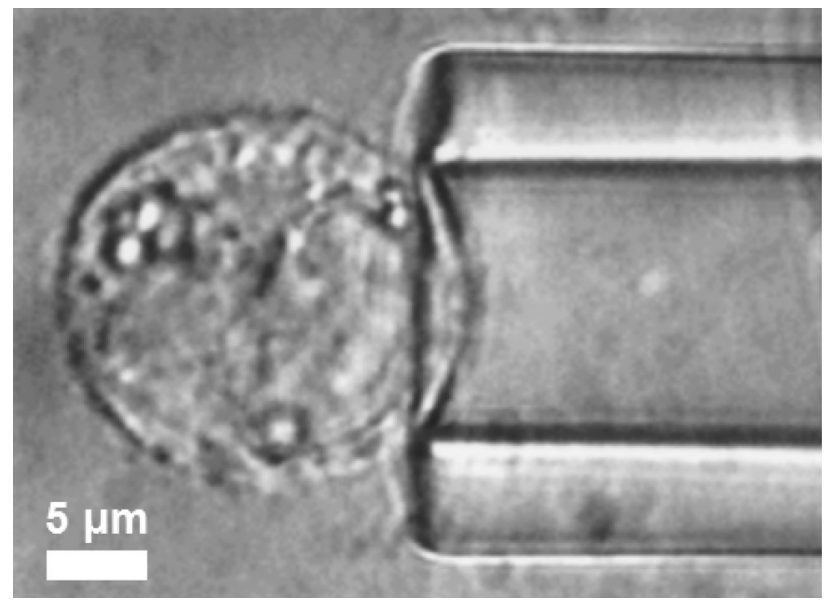

FIGURE 1. Photomicrograph of a human chondrocyte undergoing micropipette aspiration. ${ }^{15}$ method, which adopts a compressible neo-Hookean model and considers the cell as a visco-hyperelastic material. Bidhendi et al. $^{3}$ simulated the MPA of single cells by using neo-Hookean visco-hyperelastic incompressible and compressible models to study the creep behavior of cells, particularly the effect of compressibility. These previous studies demonstrated that the aspirated cell length in MPA is closely related to the compressibility of the cell and the geometric dimensions of the cell and the micropipette. Nevertheless, for the SM, a general formula that simultaneously considers cell size and compressibility has not been derived yet. In addition, the results of previous numerical

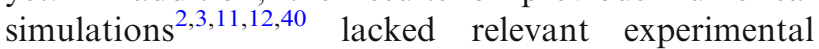
support. In our previous study, ${ }^{21}$ through theoretical and finite element analyses, we obtained an approximate elastic formula, where both cell size and compressibility are considered, to compute the aspirated length of cells in the SM. The formula could be used for reference in the accurate determination of the elastic parameters of solid-like cells by MPA.

This study aimed to verify the approximate elastic formula of the cellular aspirated length for the SM of $\mathrm{MPA}^{21}$ through experiments. For this purpose, we developed an aspiration system in which foam silicone rubber spheres with different diameters and mechanical properties were aspirated. Then, the approximate elastic formula was compared with the results of the aspiration experiment to verify the validity and accuracy of the formula.

\section{MATERIALS AND METHODS}

\section{Theoretical Analysis of the SM}

The SM considering cell size and compressibility was employed to obtain the mechanical parameters of the cells, ${ }^{21}$ wherein the cell was treated as a homogeneous and isotropic elastic body. According to elastic theory, ${ }^{34}$ in the range of linear elasticity and small deformation, the aspirated length $L_{\mathrm{s}}$, as shown in Fig. 2a, can be expressed as

$$
L_{\mathrm{s}}=\frac{f(a, b, R, v)}{E} \Delta p
$$

where $E$ and $v$ are the elastic modulus and Poisson's ratio of the cell, respectively, and $f(a, b, R, v)$ is the function of parameters $a, b, R$, and $v$.

By introducing two important geometric parameters, $\eta=(b-a) / a$ and $\xi=R / a$, we can express Eq. (1) as follows:

$$
L_{\mathrm{s}}=\frac{f^{*}(a, \eta, \xi, v)}{E} \Delta p
$$


(a)

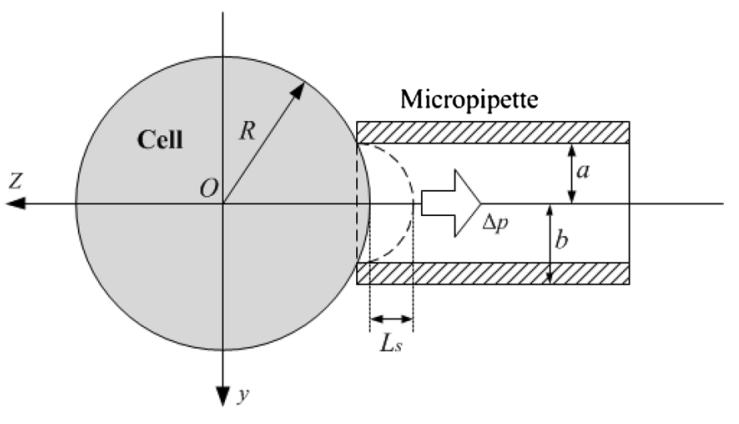

(b)

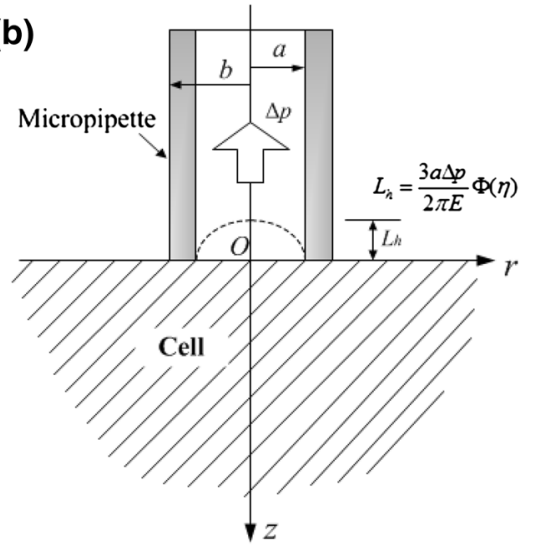

FIGURE 2. Schematic representations of the micropipette aspiration of a single cell. $a$ and $b$ are the inner and outer radii of the pipette, respectively. $\Delta p$ is the aspiration pressure. (a) Sphere model. $R$ is the radius of the cell, and $L_{\mathrm{s}}$ is the aspirated length of the model (subscript $s$ denotes sphere model). (b) Half-space model. $L_{h}$ is the aspirated length of the model (subscript $h$ denotes halfspace model), and its analytical solution was derived by Theret et al. ${ }^{33} E$ is the elastic modulus of the cell, and $\Phi(\eta)$ is the wall function of the micropipette.

However, it is very hard to derive the analytical expression of function $f^{*}(a, \eta, \xi, v)$ due to the difficulties in mathematics. It was noted that the SM degenerates into the HSM (Fig. 2b) when $\xi \rightarrow \infty$ and $v \rightarrow 0.5$. Accordingly, we assumed that Eq. (2) could be written as $L_{\mathrm{h}}$ (Fig. 2b) multiplied by a correction coefficient $\alpha$, which is a function of $\xi$ and $v$. Thus, we have the equation below:

$$
L_{\mathrm{s}}=\alpha(v, \xi) \frac{3 a \Delta p}{2 \pi E} \Phi(\eta),
$$

where $\alpha(v, \xi)$ is a hypothetic function introduced by the authors. $\Phi(\eta)$ is the wall function of the micropipette as defined previously and usually takes a value of 2.05 when $\eta$ is approximately 0.2 . Per the above analysis, $\alpha$ tends to reach 1.0 when $\xi \rightarrow \infty$ and $v \rightarrow 0.5$.

To derive the expression of function $\alpha(v, \xi)$, the finite element simulations of MPA of cell were implemented using ANSYS software (ANSYS Inc., USA, element SOLID185 for micropipette and SOLID 187 for cell). ${ }^{21}$ The micropipette was considered to be made of the borate glass $\left(E_{\mathrm{m}}=55 \mathrm{GPa}\right.$ and $v_{\mathrm{m}}=0.25$, the subscript $\mathrm{m}$ represents the micropipette). The elastic modulus $E$ of cell was set to $500 \mathrm{~Pa}$ according the previous studies, and the Poisson's ratio $v$ of the cell was adopted ranging from 0.1 to 0.499 . Parameters $a$ and $\eta$ were set to $3 \mu \mathrm{m}$ and 0.2 respectively, and the radius of cell $R$ was changed from 6 to $45 \mu \mathrm{m}$, i.e. the range of parameter $\xi$ was $2-15$. The sliding contact condition between the bottom of micropipette and the surface of cell was considered (element TARGE170 for micropipette and CONTA174 for cell). At a given aspiration pressure $(\Delta p=25 \sim 100 \mathrm{~Pa})$, the aspirated length of cell $L_{\mathrm{s}}$ with the different $\xi$ and $v$ were simu- lated. Then, a series of discrete values of function $\alpha$ were calculated as follows:

$$
\alpha(v, \xi)=\frac{2 \pi E}{3 a \Delta p \Phi(\eta)} L_{\mathrm{s}} .
$$

The concrete form of function $\alpha$ should be given before fitting the numerical results from Eq. (4). According to the trend of inverse proportion between $\alpha$ and $\xi$ in the simulation, a Hill's function was adopted by which Boudou et al. ${ }^{4}$ characterized the relationship of aspirated length and the normalized thickness successfully in the MPA of thin non-adherent soft tissue. The relation between $\alpha$ and $v$ can refer to the elastic theory on the problem of "Load Distributed over a Part of the boundary of a Semi-infinite Solid" ${ }^{34}$ where the maximum displacement was proportional to the term $\left(1-v^{2}\right)$. Accordingly, we assumed the form of the function $\alpha(v, \xi)$ as follows:

$$
\alpha(v, \xi)=A\left(1+\frac{B}{\xi^{C}}\right)\left(1-v^{2}\right),
$$

where $B$ and $C$ are the dimensionless parameters to be determined by fitting the simulation data. Parameter $A$ was set to 1.33 to ensure the term ' $A\left(1-v^{2}\right)$ ' tends to 1.0 when $v$ tends to $0.5 .^{21}$ Very satisfactory fits were obtained, as shown in Fig. 3.

Therefore, the approximate elastic formula of the aspirated length for the SM can be expressed as follows:

$$
L_{\mathrm{s}}=A\left(1+\frac{B}{\xi^{C}}\right)\left(1-v^{2}\right) \frac{3 a \Delta p}{2 \pi E} \Phi(\eta) .
$$

Obviously, Eq. (6) is an empirical expression obtained by theoretical and numerical analysis and lacks 


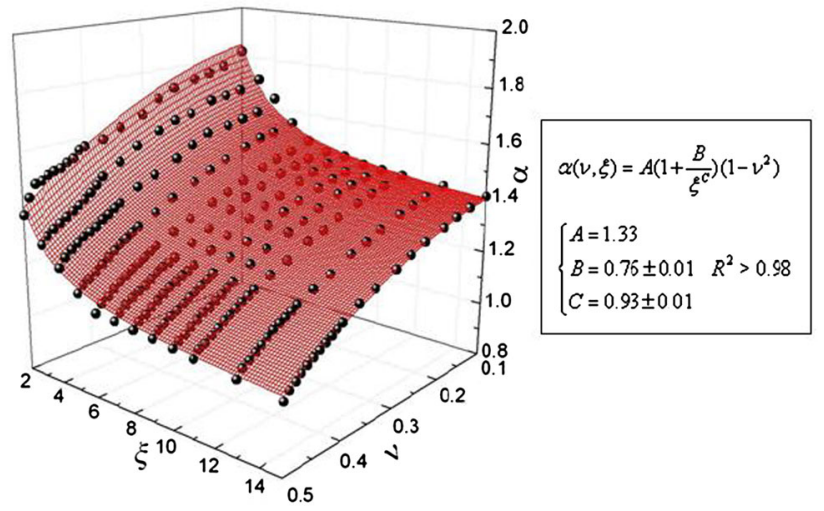

FIGURE 3. Surface fitting of the simulation data. ${ }^{21}$ To satisfy the characteristics of $\alpha(v, \xi)$, parameter $A$ was set to 1.33 . B and $C$ are the fitting parameters.

experimental support. Next, the formula would be validated using a self-developed experimental system.

\section{Aspiration System for Spheres}

An aspiration system was developed to verify Eq. (6). The system is composed of three parts: a pressure supply system (PSS), an experiment box (EB), and a measurement system (MS). The aspiration system is shown in Fig. 4a.

The PSS mainly consisted of a hand pressure pump (SB-2.5, China) and a one-way valve. The one-way valve allowed water to flow from the pump to the vessel and prohibited reverse flow. Consequently, the pressure in the vessel was constant. The EB was a steel-sealed vessel with a thin tube leading to the outside at the bottom. For consistency with the choice of wall parameter $\eta$ for the value of wall function $\Phi(\eta)$ in Eq. (6) as 2.05, the inner and outer diameters of the tube were 1.0 and $1.2 \mathrm{~cm}$, respectively. A pressure sensor and a one-way valve were connected with the EB from the two sides, respectively. The pressure in the EB was exerted through the PSS after placing the spherical specimen on the tube and filling the EB with water. As the pressure inside the EB was higher than that outside $(\sim 0.1 \mathrm{MPa})$, the specimen was aspirated into the tube similarly to cell deformation during MPA. The MS consisted of a pressure sensor (PTG503-1000K-10V0.5-M20-3m, China), a laser displacement sensor (optoNCDT1605, Germany), and a dynamic collection system (DH5923, China). The inner pressure $(\Delta p)$ and the deformation $(\Delta L)$ of the spherical specimen were measured using the pressure sensor and the laser displacement sensor, respectively. The data were then collected and analyzed via the dynamic collection and analysis system.

\section{Foam Silicone Rubber}

Foam silicone rubber is a spongy-like elastomer prepared by foaming and curing the silicone rubber base at room temperature. Six types of foam silicone rubber were labeled 1-6 according to porosity from large to small (where 6 is solid), and their basic physical parameters are listed in Table 1 .

Standard tests for stretch and compression $(\mathrm{GB} / \mathrm{T}$ 528-2009 and GB/T 7757-2009) were carried out at room temperature using INSTRON 5544 (the United States, INSTRON Company) to measure the mechanical parameters of the foam silicone rubber. The maximum strain was controlled at less than $30 \%$. As the following aspiration experiment was conducted in water, the specimens were immersed in tap water at room temperature for $24 \mathrm{~h}$ and then dried before being tested again.

To verify the relationship among the aspirated length $L$, the geometric parameter $\xi$, and the Poisson's ratio $v$ in Eq. (6), spherical specimens with eight diameters ranging from 1.5 to $5.0 \mathrm{~cm}$ were constructed from the six types of foam silicone rubber indicated in Table 1, as shown in Fig. 5.

\section{Aspiration of the Foam Silicone Rubber Spheres}

First, we smeared some lubricant on the nozzle of the suction tube at the bottom of EB and placed a spherical specimen on the nozzle. The type and size of the sample were recorded beforehand. Then, we sealed the EB and balanced and cleared each channel of the acquisition and analysis system. Pressure was applied by water injection to the EB via the hand pump, which caused an elastic aspiration of a portion of the spherical sample. The pressure was increased incrementally (increment was approximately $0.05 \mathrm{MPa}$ ) and was usually maintained for $10 \mathrm{~s}$ until a steady state was 
(a)

Dynamic signal

Hand pressure pump

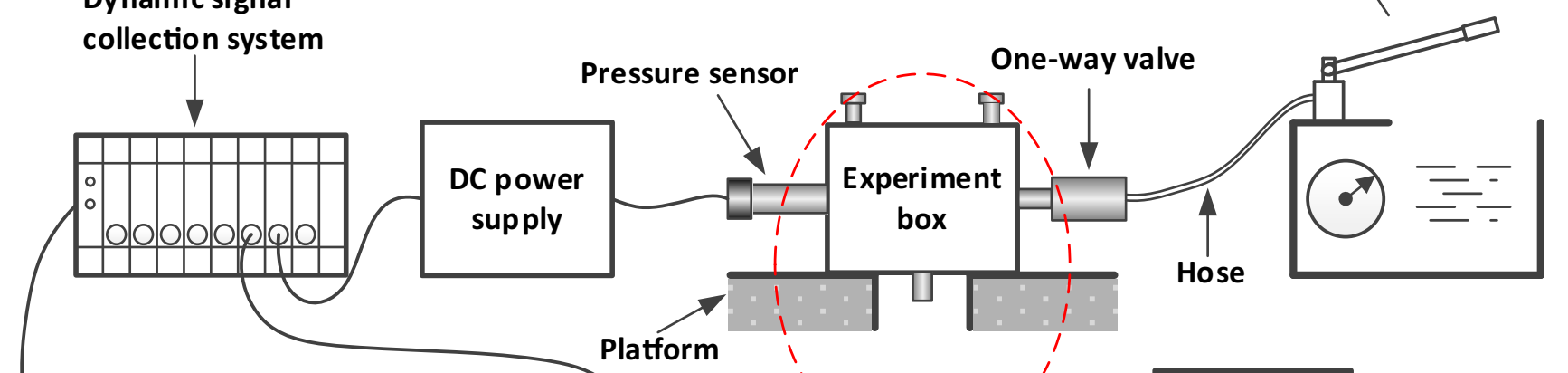

(b)

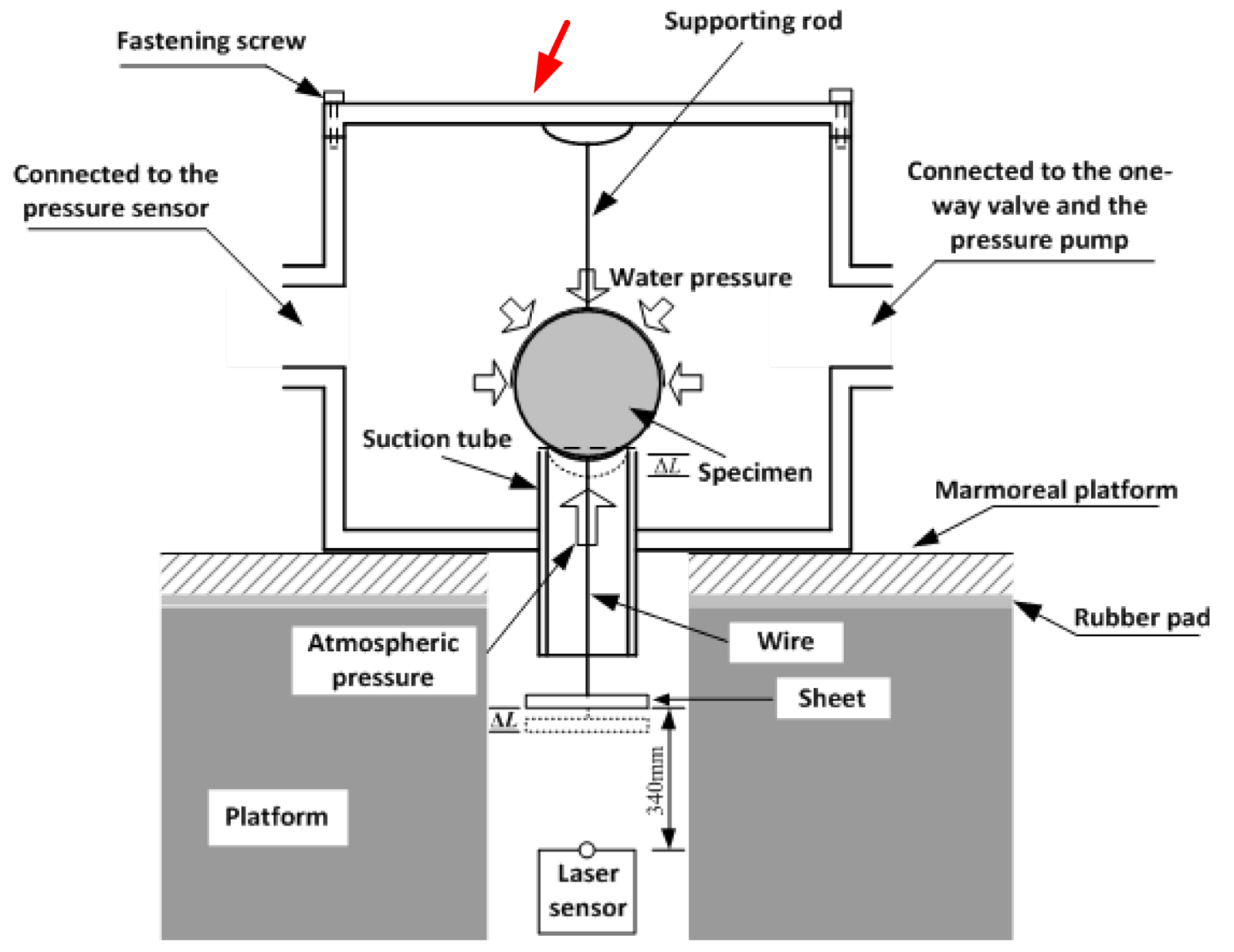

FIGURE 4. (a) Diagram of the aspiration system for spheres. (b) Cross-sectional structure of the EB. The gauge of the laser displacement sensor is $340 \mathrm{~mm}$.

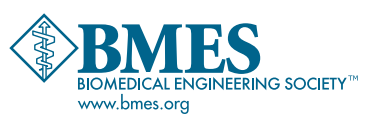


TABLE 1. Physical parameters for each type of foam silicone rubber.

\begin{tabular}{lcccccc}
\hline Type & 1 & 2 & 3 & 4 & 5 & 6 \\
\hline Porosity $(\%)$ & 58.0 & 43.5 & 32.7 & 20.9 & 0 \\
Density $\left(\mathrm{g} / \mathrm{cm}^{3}\right)$ & 0.55 & 0.63 & 0.77 & 0.92 & 12.8 & 1.05 \\
\hline
\end{tabular}

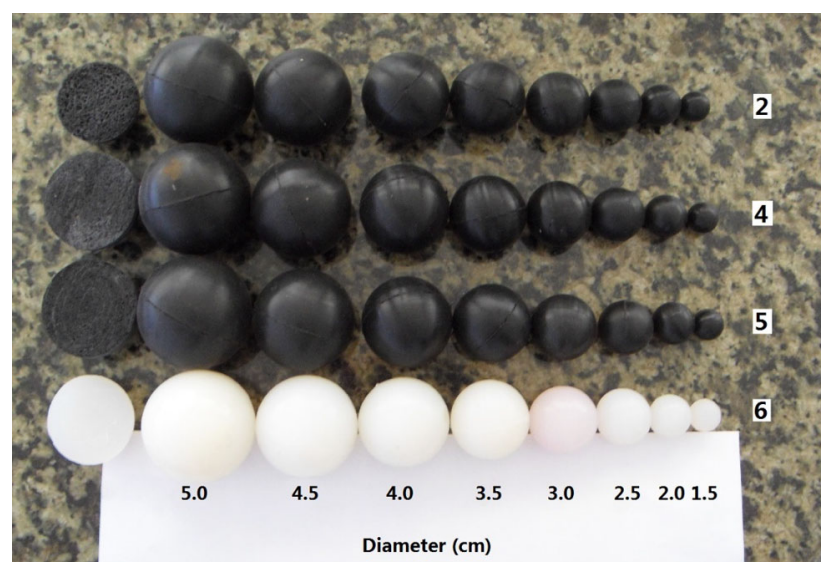

FIGURE 5. Different diameters of spherical samples made of foam silicone rubber.

reached. Since the range of the pressure sensor was $1 \mathrm{MPa}$, the maximum pressure was controlled to $0.9 \mathrm{MPa}$. The profile of EB is shown in Fig. $4 \mathrm{~b}$. To measure the displacement of the bottom of the specimen conveniently, one end of a hard and straight wire was fixed on the bottom of the sample with a sucker, and the other end was connected to a smooth sheet perpendicularly, as shown in Fig. 4b. Thus, when the specimen was displaced downward, the sheet would produce synchronous and equivalent movement, and then the laser sensor would measure the displacement of the sheet. In addition, a telescopic rod was designed, considering that the density of the foam silicone rubber was less than that of water. To one end of the rod was adsorbed a sealing cover, and the other end held the specimen slightly. Accordingly, the sample could be fixed on the top of the tube and only produce downward deformation because of the confined compression. The data of pressure and displacement were automatically collected by the software.

\section{RESULTS}

\section{Mechanical Parameters of the Foam Silicone Rubber}

The standard experiments indicated that the tensile and compressive stiffness of the different types of foam silicone rubber significantly decrease with increasing porosity. For each type, the tensile stiffness is generally lower than that of compression. However, the above difference gradually disappears with increasing porosity, which is consistent with the previous studies. ${ }^{14,17,37}$ The true stress-strain curves of uniaxial stretch and compression for Types 1-6 are shown in Fig. 6.

In addition, the mechanical properties of the material during stretch and compression were not significantly changed after $24 \mathrm{~h}$ of water immersion (WI). The true stress-strain curves of stretch and compression of Types 4 and 6 before and after WI are shown in Fig. 7.

The elastic modulus of the material was calculated by taking the average value of the tensile and compression moduli, which were derived using linear fitting of the true stress-strain data in the range of small strain $(\varepsilon<0.15)$ before WI. The Poisson's ratio of the material was obtained by taking the absolute value of the ratio of transverse strain to the longitudinal strain. The elastic parameters of the foam silicone rubber are listed in Table 2.

\section{Results of the Aspiration of Foam Silicone Rubber Spheres}

For the aspiration of spheres, the inner diameter of the tube was $1.0 \mathrm{~cm}$, and the diameters of the spherical specimens ranged from 1.5 to $5.0 \mathrm{~cm}$. Hence, the range of the geometric parameter $\xi$ (specimen radius/inner tube radius) was $1.5-5.0$, which is generally consistent with the $\xi$ of MPA of cells (1.48-2.58 for normal chondrocytes, and 1.46-2.38 for osteoarthritis chondrocytes) ${ }^{38}$ The aspiration results of the specimens with different $\xi$ and mechanical properties are shown in Fig. 8.

Regardless of the type of material, the aspiration length $L$ decreased with increasing parameter $\xi$, which was consistent with the approximate elastic formula Eq. (6). The experimental results for Types 1 and 2 were close to those obtained using Eq. (6), as shown in Figs. $8 \mathrm{a}$ and $8 \mathrm{~b}$. For the Type 2, the coefficient of determination (COD) $R^{2}$ between the experiment results and Eq. (6) for $\xi=1.5$ and 3 were 0.99 and 0.98 respectively. Whereas the results of experiment for Types 5 and 6 were slightly larger than that of Eq. (6), as shown in Figs. 8c and 8d. $R^{2}$ of $\xi=1.5$ and 4 for Type 6 were 0.94 and 0.95 .

To validate the relationship between the aspiration length $L$ and Poisson's ratio $v$ in Eq. (6), the normal- 

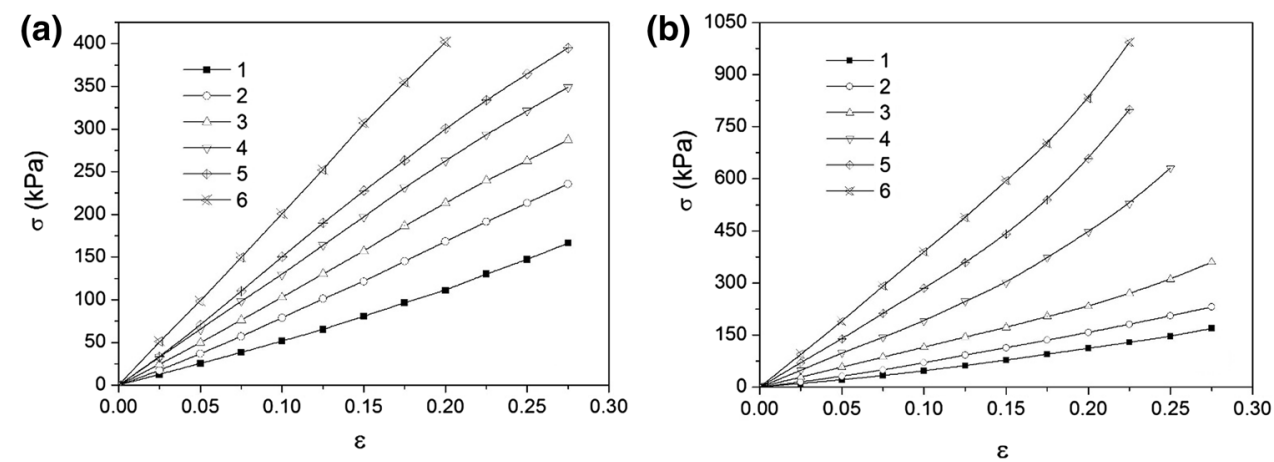

FIGURE 6. Uniaxial true stress-strain curves of six types of foam silicone rubber obtained by standard tests. (a) stretch; (b) compression.
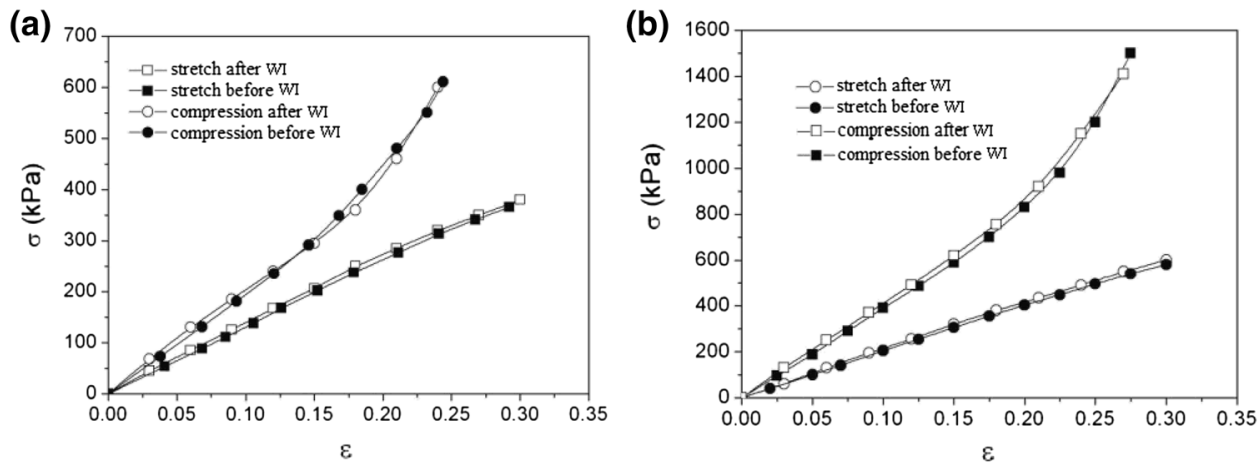

FIGURE 7. True stress-strain curves of foam silicone rubber before and after WI. (a) Type 4; (b) Type 6.

TABLE 2. Elastic moduli and Poisson's ratios for six types of foam silicone rubber $(n=10$, mean \pm SD).

\begin{tabular}{lcccccc}
\hline Type & 1 & 2 & 3 & 4 & 5 & 6 \\
\hline$E(\mathrm{MPa})$ & $0.51 \pm 0.12$ & $0.76 \pm 0.09$ & $0.95 \pm 0.05$ & $1.46 \pm 0.08$ & $2.05 \pm 0.22$ & $2.95 \pm 0.16$ \\
$v$ & $0.18 \pm 0.13$ & $0.24 \pm 0.11$ & $0.31 \pm 0.09$ & $0.38 \pm 0.05$ & $0.44 \pm 0.03$ & $0.49 \pm 0.01$ \\
\hline
\end{tabular}

ization was performed for the aspirated length and the negative pressure of the experiments using the radius of the tube and elastic modulus respectively, as shown in Fig. 9.

From the figures, we can see that the aspirated length $L$ decreased with increasing Poisson's ratio $v$ for the same $\xi$. This result was very close to that of the approximation formula Eq. (6) $\left(R^{2}=0.99,0.98\right.$ and 0.98 for Types 2, 4 and 6 respectively). Thus, the relationship between the aspiration length $L$ and the Poisson's ratio $v$ during the MPA can be reflected objectively by Eq. (6).

\section{DISCUSSION}

In this paper, an aspiration system, in which spherical specimens with different diameters and mechanical properties were aspirated similarly to the cells under MPA, was developed to verify the approximate elastic formula Eq. (6) for the cellular aspirated length of our previously developed SM during MPA. Comparisons indicated that the predictions obtained using Eq. (6) agreed with the experimental results. Therefore, it is confirmed that Eq. (6) can be applied to characterize the aspiration response of the elastic sphere in the condition of linear elasticity and small deformation.

It should be noted that, in the derivation of Eq. (6) obtained by theoretical analysis and numerical simulations, there was no geometric scale limitation introduced. Theoretically, Eq. (6) is applicable for any geometric scale when the vital geometric parameter $\xi$ is greater than 1.0 in the case of linear elasticity and small deformation. Therefore, the formula can be applied to the MPA of cells, and is also suitable for the aspiration of elastic spheres on macro scales. In this study, the formula is proved to be valid on a macroscopic scale, thus, it

\footnotetext{
mechanical properties were aspirated similarly to the$$
\text { chanical properties were aspirated similarly to the }
$$ 

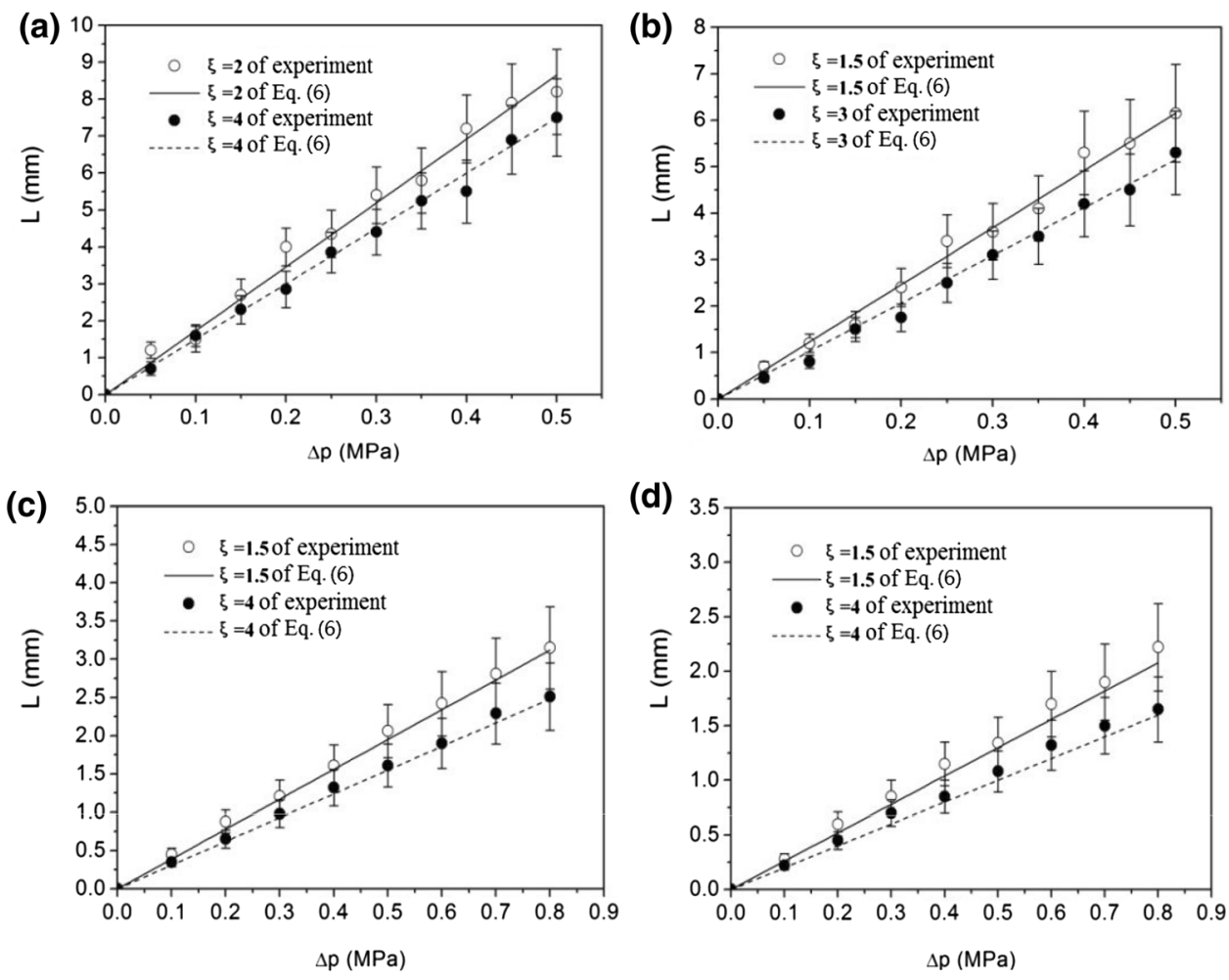

FIGURE 8. Aspiration responses of the spherical samples with different $\xi$ and mechanical properties $(n=5)$. (a) Type 1; (b) Type 2; (c) Type 5; (d) Type 6.
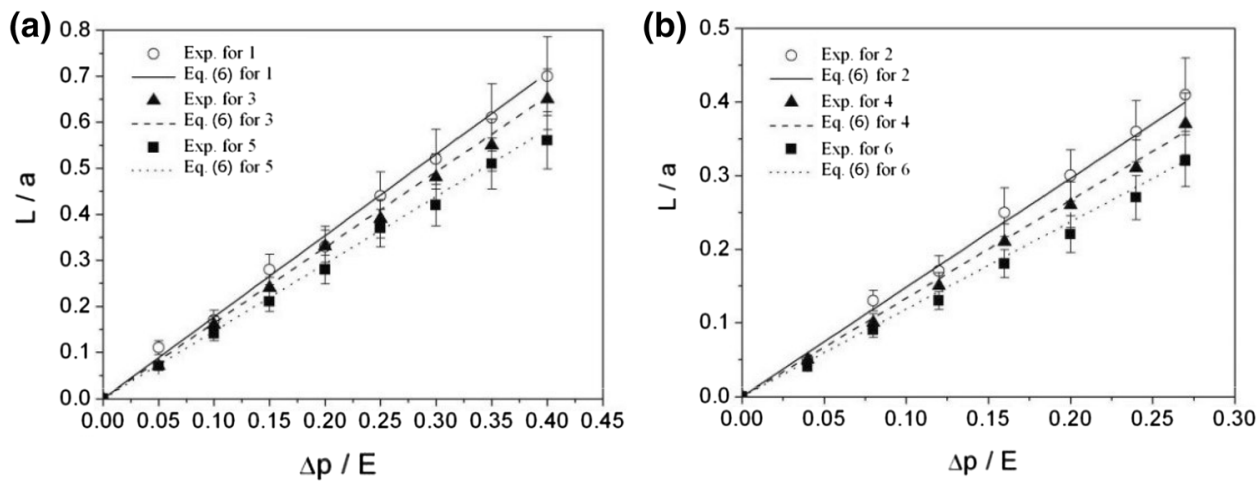

FIGURE 9. Normalized curves of the aspiration responses for the specimens of foam silicone rubber $(n=5)$. (a) $\xi=2$ for Types 1 , 3 and 5; (b) $\xi=4$ for Types 2, 4 and 6.

can be concluded that Eq. (6) can also capture the elastic behavior of the cells during MPA objectively under linear conditions, and could be applied to estimate the elastic parameters of cells more accurately. Moreover, the numerical results indicated that $L_{\mathrm{s}}$ was proportional to $\Delta p$ and inversely proportional to $E$ (as shown in Fig. 10). ${ }^{20}$ The proportional relationship between $L_{\mathrm{s}}$ and $a$ can also be confirmed in the same way. Thus, the value of function $\alpha$ was independent of $a, \Delta p$ and $E$.

Therefore, the values of parameters $A, B$ and $C$ depend only on the form of function $\alpha$, and are un- iquely determined in the given form of function in our previous study.

To evaluate the error of elastic moduli derived from the HSM and SM, another parameter $e$ was introduced and is defined as follows ${ }^{21}$ :

$$
e=\left|\frac{E_{\mathrm{h}}-E_{\mathrm{s}}}{E_{\mathrm{s}}}\right|
$$

where $E_{\mathrm{h}}$ and $E_{\mathrm{s}}$ are the elastic moduli obtained from the HSM and SM, respectively. From the study of 


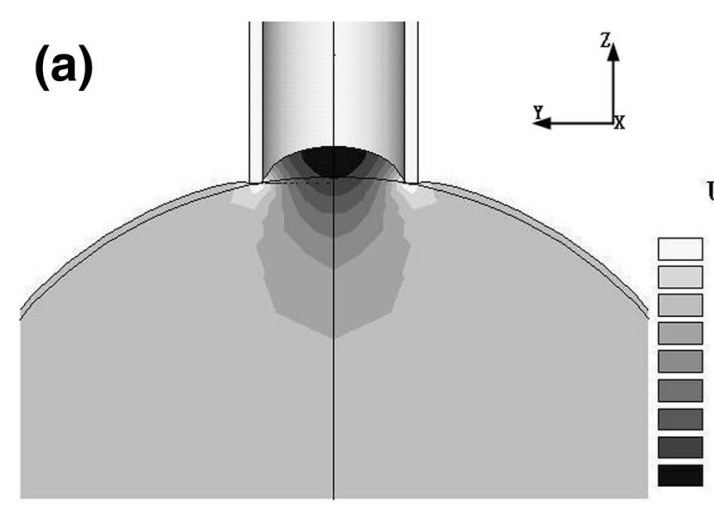

$\mathrm{Uz}$

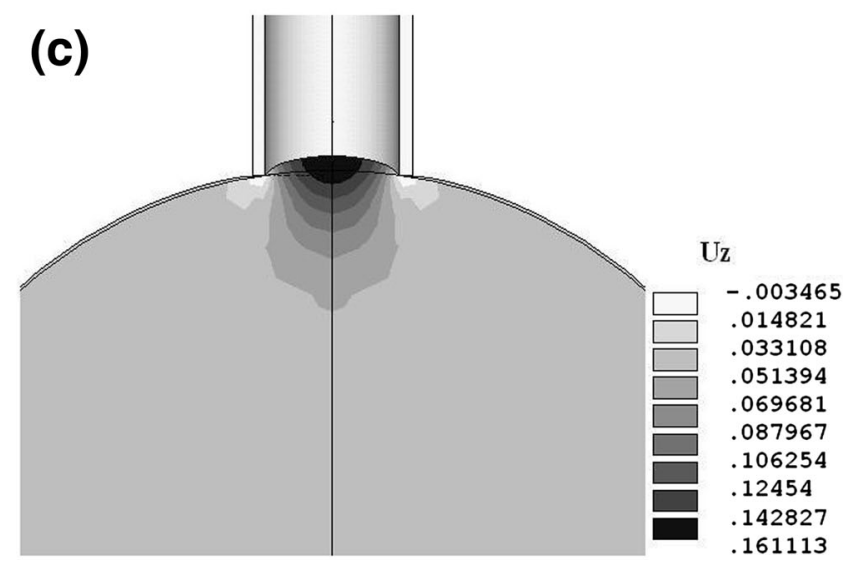

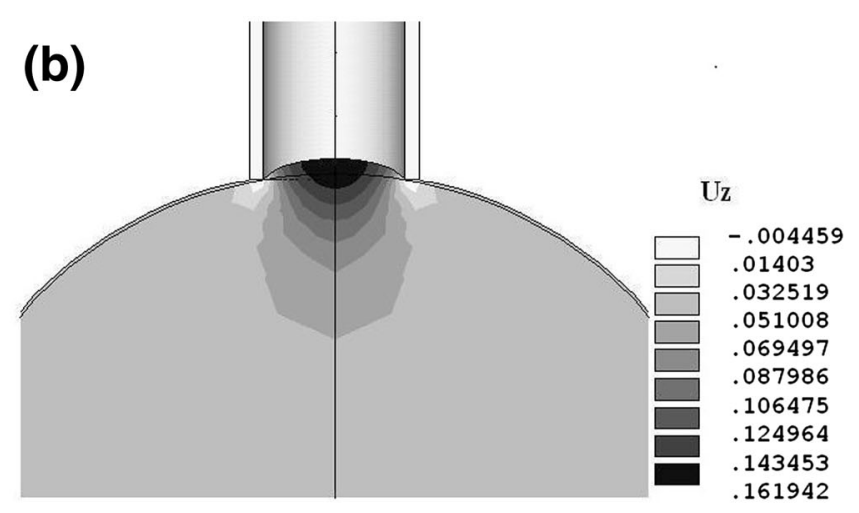

(d)

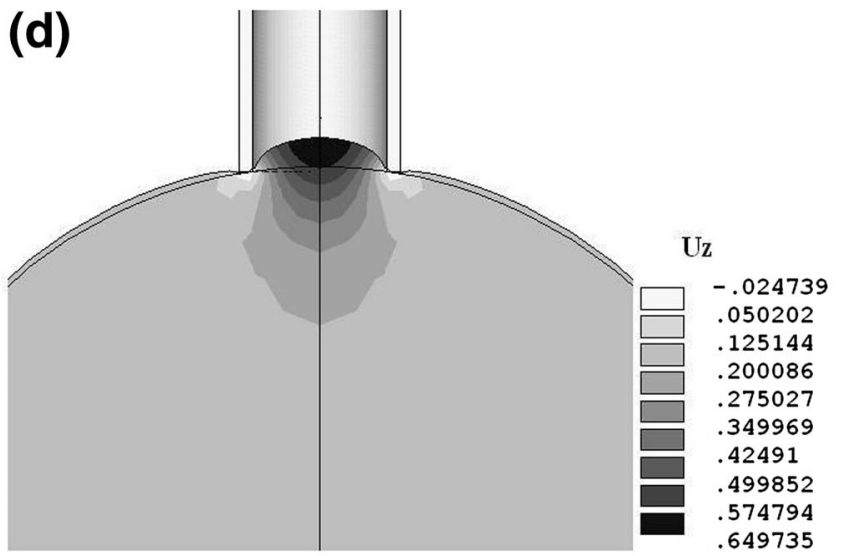

FIGURE 10. Contour plots of the aspirated displacement of cell corresponding to different $\Delta p$ and $E$ obtained using ANSYS $(v=0.499, a=3 \mu \mathrm{m})$. (a) $\xi=6, \Delta p=50 \mathrm{~Pa}, E=250 \mathrm{~Pa}$; (b) $\xi=6, \Delta p=50 \mathrm{~Pa}, E=1000 \mathrm{~Pa}$; (c) $\xi=7, E=500 \mathrm{~Pa}, \Delta p=25 \mathrm{~Pa}$; (d) $\xi=7, E=500 \mathrm{~Pa}, \Delta p=100 \mathrm{~Pa}$. Scale factor is 2.0 for (b) and (c).

Theret et $_{\text {al. }}{ }^{33}$ and Eq. (6), $E_{\mathrm{h}}$ and $E_{\mathrm{s}}$ can be expressed as follows:

$$
\begin{gathered}
E_{\mathrm{h}}=\frac{3 a \Delta p \Phi(\eta)}{2 \pi L}, \\
E_{\mathrm{s}}=\alpha(v, \xi) \frac{3 a \Delta p \Phi(\eta)}{2 \pi L},
\end{gathered}
$$

where $L$ is the aspirated length of the cells in the MPA experiment, and the other parameters are the same as those in Eq. (6). In the incompressible case of Eq. (6) $(v=0.5)$, when $\xi=1.43$, $e$ is equal to $35.5 \%$, which is consistent with the corresponding $35 \%$ obtained by Haider et al. ${ }^{12}$ using the boundary element method. For the compressible cases, the comparisons between the results of this study and those of Baaijens et al. ${ }^{2}$ are shown in Fig. 11. The parameter $\xi$ markedly affected the aspirated length, and the approximation formula agreed with the results given above, particularly within the range of small deformation (Fig. 11a). Although the results were derived from a compressible hyperelastic model, they were consistent with the approxi- mate elastic formula Eq. (6) within the scope of small deformation (Fig. 11b).

Combined with the experimental data of MPA for rabbit chondrocytes, ${ }^{38}$ the differences in the elastic parameters derived from the HSM, incompressible sphere model (ICSM), and compressible sphere model (CSM) were investigated. Notably, the Poisson's ratio of the chondrocytes is unknown here. Therefore, to determine $E$ in the compressible cases, $v$ was taken to be $0.2,0.3$, and 0.4 , as in previous studies. ${ }^{3,5,15} \mathrm{In}$ addition, another concern is the bulk modulus $K$ of the cell, and it is defined as follows:

$$
K=\frac{E}{3(1-2 v)} .
$$

$E$ and $K$ obtained from the different models are shown in Table 3.

The elastic parameters derived from the different models significantly differed. In consideration of the parameter $\xi$, the elastic modulus $E$ of ICSM was 1.48 times that of HSM. Furthermore, $E$ of CSM when $v$ was $0.2,0.3$, or 0.4 was $1.89,1.79$, or 1.65 times as large as that of HSM, respectively. We also observed that 

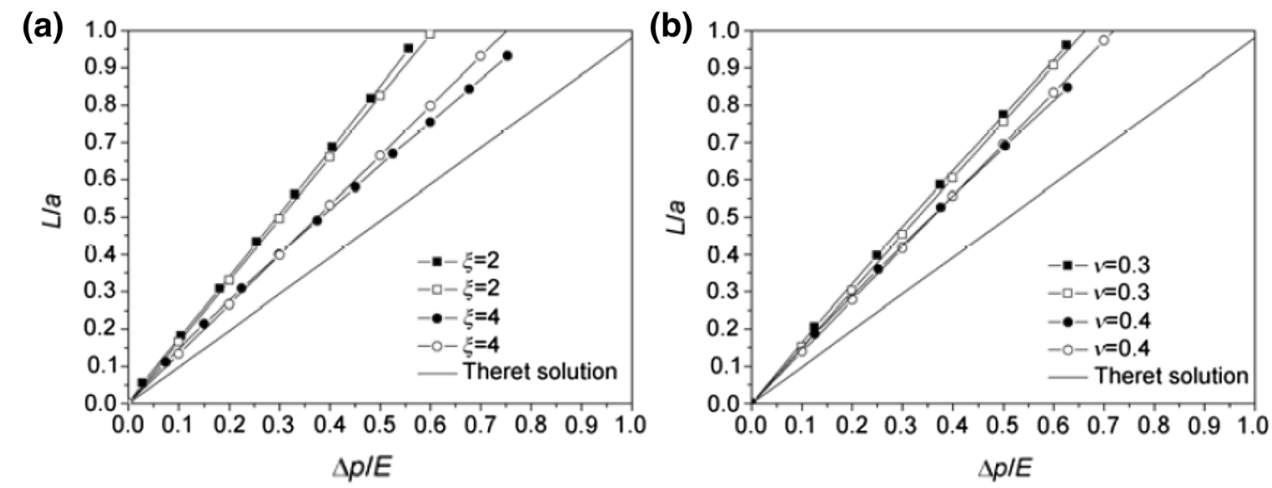

FIGURE 11. Normalized aspirated length $L a$ as a function of the normalized aspiration pressure $\Delta p / E$. The lines with the closed symbols correspond to the results of Baaijens et al., ${ }^{2}$ whereas the lines with the open symbols correspond to Eq. (6). (a) Results of compressible elastic model with $v=0.4$; (b) results of $\xi=3$ (the lines with closed symbols were derived from a compressible neoHookean material model by Baaijens et al. ${ }^{2}$ ).

TABLE 3. Elastic parameters of normal rabbit chondrocytes derived from different models. (Mean \pm SD, $n=51$ ).

\begin{tabular}{lllr}
\hline Model & & $E(\mathrm{KPa})$ & $K(\mathrm{KPa})$ \\
\hline HSM & & $0.5690 \pm 0.4292$ & $+\infty$ \\
ICSM & & $0.8410 \pm 0.6560$ & $+\infty$ \\
CSM & $v=0.2$ & $1.0737 \pm 0.8376$ & $0.5965 \pm 0.4654$ \\
& $v=0.3$ & $1.0178 \pm 0.7940$ & $0.8482 \pm 0.6617$ \\
& $v=0.4$ & $0.9395 \pm 0.7329$ & $1.5659 \pm 1.2216$ \\
\hline
\end{tabular}

the value of Poisson's ratio $v$ in the range of $0.2-0.4$ had little effect on $E$. When $v$ was $0.4, E$ dropped by $12 \%$ compared with that when $v$ was 0.2 . However, the changes of parameter $v$ can dramatically influence the determination of bulk modulus $K$, which, when $v=0.4$, was 2.63 times that when $v=0.2$. The sensitivity of $K$ to $v$ may significantly affect the subsequent viscoelastic analysis, which will be presented in detail in future studies.

The determination of the mechanical parameters of foam silica rubber is an important part of this study. Essentially, foam silicone rubber is porous, hyperelastic, and not strictly homogeneous. For simplification, the rubber was treated as a homogeneous isotropic elastic continuous medium and was tested for the determination of the elastic modulus and Poisson's ratio by standard tests. Figure 8 shows that with decreasing porosity, the predictions of Eq. (6) gradually deviated from the experimental values and became less than the experimental values. Such observations may be attributed to the adoption of the average elastic moduli of stretch and compression in the approximate formula. Although a certain degree of compression existed at the contact region of the end of the micropipette, the mechanical properties of stretch were considered to play a crucial role during MPA. As the mechanical properties differed greatly under stretch and compression for Types 5 and 6 (Fig. 6), the average modulus adopted was relatively larger than the tensile modulus. Thus, the results of the approximate formula were smaller than the experimental results (Figs. 8c and 8d). For Types 1 and 2, the experimental results agreed with the approximate formula (Figs. 8a and $8 b$ ) as a result of the close mechanical properties in stretch and compression (Fig. 6).

In summary, an aspiration system of spheres was developed in this study to verify the approximate elastic formula Eq. (6) of the aspirated length of a single cell for our previously developed SM during MPA. Compared with the results of sphere aspiration experiments and the studies of other groups, the approximate formula was confirmed within the range of linear elasticity and small deformation. Thus, we conclude that the formula is valid for characterizing the elastic responses of solid-like cells in MPA experiments. Furthermore, comparisons of the elastic parameters derived from the HSM, ICSM, and CSM indicate that parameters $\xi$ and $v$ exert marked effects on the determination of $E$ and $K$. Thus, these parameters should be considered when determining the cellular mechanical properties using MPA.

Finally, it should be noted that the aspiration length of solid-like cells is essentially time dependent. ${ }^{2,30,39}$ After an initial elastic response proportional to the 
aspiration pressure, a creep response was observed before the aspiration length, and the cell shape reached a steady state. Both the initial response and the final steady state may be described using an elastic constitutive model. This paper focused only on the elastic responses of cells, which accounted for the transient behavior of the cell under negative pressure. Under the premise of linear constitutive relation and small deformation, the mechanical behaviors of material in the viscoelastic phase-space could be derived from its elastic responses by the corresponding principle of elasticity to viscoelasticity. Thus, the viscoelastic behaviors of a single solid-cell during MPA considering the size and compressibility of cell could be further studied based on the elastic responses obtained in our previous study, and will be presented in future work.

\section{ACKNOWLEDGMENTS}

Supports from the National Natural Science Foundation of China (Grant Nos. 11572213, 11632013, 11702184 and 11472185), the Natural Science Foundation of Shanxi Province, China (Grant No. 2014021013), and the Youth Funds of Taiyuan University of Technology (No. 2013T079) are acknowledged.

\section{CONFLICT OF INTEREST}

None of the authors have any competing financial interests related to this paper.

\section{REFERENCES}

\footnotetext{
${ }^{1}$ Ahmad, I. L., and M. R. Ahmad. Trends in characterizing single cell's stiffness properties. Micro Nano Syst. Lett. 2:8, 2014. https://doi.org/10.1186/s40486-014-0008-5.

${ }^{2}$ Baaijens, F. P. T., W. R. Trickey, T. A. Laursen, and F. Guilak. Large deformation finite element analysis of micropipette aspiration to determine the mechanical properties of the chondrocyte. Ann. Biomed. Eng. 33:494-501, 2005.

${ }^{3}$ Bidhendi, A. J., and R. K. Korhonen. A finite element study of micropipette aspiration of single cells: Effect of compressibility. Comput. Math. Methods Med. 2012. http s://doi.org/10.1155/2012/192618.

${ }^{4}$ Boudou, T., J. Ohayon, Y. Arntz, G. Finet, C. Picart, and P. Tracqui. An extended modeling of the micropipette aspiration experiment for the characterization of the Young's modulus and Poisson's ratio of adherent thin biological samples: numerical and experimental studies. $J$. Biomech. 39:1677-1685, 2006.

${ }^{5}$ Charras, G. T., P. P. Lehenkari, and M. A. Horton. Atomic force microscopy can be used to mechanically
}

stimulate osteoblasts and evaluate cellular strain distributions. Ultramicroscopy 86:85-95, 2001.

${ }^{6}$ Chaudhuri, O., and D. J. Mooney. Stem-cell differentiation: anchoring cell-fate cues. Nat. Mater. 11:568-569, 2012.

${ }^{7}$ Fung, Y. C. Biomechanics: Mechanical Properties of Living Tissues. New York: Springer, p. 788, 1993.

${ }^{8}$ Galbraith, C. G., and M. P. Sheetz. Forces on adhesive contacts affect cell function. Curr. Opin. Cell Biol. 10:566571, 1998.

${ }^{9}$ Guilak, F., G. Erickson, and H. Ting-Beall. The effects of osmotic stress on the viscoelastic and physical properties of articular chondrocytes. Biophys. J. 82:720-727, 2002.

${ }^{10} \mathrm{Guz}$, N., M. Dokukin, V. Kalaparthi, and I. Sokolov. If cell mechanics can be described by elastic modulus: study of different models and probes used in indentation experiments. Biophy. J. 107:564-575, 2014.

${ }^{11}$ Haider, M. A., and F. Guilak. An axisymmetric boundary integral model for incompressible linear viscoelasticity: application to the micropipette aspiration contact problem. ASME J. Biomech. Eng. 122:236-244, 2000.

${ }^{12}$ Haider, M. A., and F. Guilak. An axisymmetric boundary integral model for assessing elastic cell properties in the micropipette aspiration contact problem. ASME J. Biomech. Eng. 124:586-595, 2002.

${ }^{13}$ Hochmuth, R. M. Micropipette aspiration of living cells. $J$. Biomech. 33:15-22, 2000.

${ }^{14} \mathrm{Hu}$, W. J., H. Chen, K. Zhang, and X. Chen. Effect of porosity on the properties of open cell silicone rubber foam materials. China Rubber Ind. 45:647-651, 1998; ((In Chinese)).

${ }^{15}$ Jones, W. R., H. P. Ting-Beall, G. M. Lee, S. S. Kelly, R. M. Hochmuch, and F. Guilak. Alterations in the Young's modulus and volumetric properties of chondrocytes isolated from normal and osteoarthritic human cartilage. $J$. Biomech. 32:119-127, 1999.

${ }^{16}$ Khani, M.-M., M. Tafazzoli-Shadpour, Z. Goli-Malekabadi, and N. Haghighipour. Mechanical characterization of human mesenchymal stem cells subjected to cyclic uniaxial strain and TGF- $\beta 1$. J. Mech. Behav. Biomed. 43:1825, 2015.

${ }^{17}$ Kinney, J. H., G. W. Marshall, S. J. Marshall, and D. L. Haupt. Three-dimensional imaging of large compressive deformations in elastomeric foams. J. Appl. Polym. Sci. 80:1746-1755, 2001.

${ }^{18}$ Lee, G. Y. H., and C. T. Lim. Biomechanics approaches to studying human diseases. Trends Biotechnol. 25:111-118, 2007.

${ }^{19}$ Lekka, M., and P. Laidler. Applicability of AFM in cancer detection. Nat. Nanotechnol. 4:72-73, 2009.

${ }^{20} \mathrm{Li}$, Y. S. Study on the mechanical models for micropipette aspiration of cells. Thesis for the Doctor Degree of Taiyuan University of Technology, pp. 51-52, 2014 (In Chinese).

${ }^{21}$ Li, Y. S., and W. Y. Chen. Finite element analysis of micropipette aspiration considering finite size and compressibility of cells. Sci. China Phys. Mech. 56:2208-2215, 2013.

${ }^{22} \mathrm{Li}$, J., M. Dao, and S. Suresh. Spectrin-level modeling of the cytoskeleton and optical tweezers stretching of the erythrocyte. Biophys. J. 88:3707-3719, 2005.

${ }^{23}$ Loh, O., A. Vaziri, and H. D. Espinosa. The potential of MEMS for advancing experiments and modeling in cell mechanics. Exp. Mech. 49:105-124, 2010.

${ }^{24}$ Maksym, G. N., B. Fabry, J. P. Butler, D. Navajas, D. J. Tschumperlin, J. D. Laporte, and J. J. Fredberg. Mechanical properties of cultured human airway smooth 
muscle cells from 0.05 to $0.4 \mathrm{~Hz}$. J. Appl. Physiol. 89:16191632, 2000.

${ }^{25}$ Mills, J. P., L. Qie, M. Dao, C. T. Lim, and S. Suresh. Nonlinear elastic and viscoelastic deformation of the human red blood cell with optical tweezers. Mech. Chem. Biosyst. 1:169-180, 2004.

${ }^{26}$ Nash, G. B., E. O’Brien, and J. A. Dormandy. Abnormalities in the mechanical properties of red blood cells caused by Plasmodium falciparum. Blood. 74:855-861, 1989.

${ }^{27}$ Pachenari, M., S. M. Seyedpour, M. Janmaleki, S. B. Shayan, S. Taranejoo, and H. Hosseinkhani. Mechanical properties of cancer cytoskeleton depend on actin filaments to microtubules content: investigating different grades of colon cancer cell lines. J. Biomech. 47:373-379, 2014.

${ }^{28}$ Paszek, M. J., N. Zahir, and V. M. Weaver. Tensional homeostasis and the malignant phenotype. Cancer Cell. 8:241-254, 2005.

${ }^{29}$ Roduit, C., S. Sekatski, G. Dietler, S. Catsicas, F. Lafont, and S. Kasas. Stiffness to mography by atomic force microscopy. Biophys. J. 97:674-677, 2009.

${ }^{30}$ Sato, M., D. P. Theret, L. T. Wheeler, N. Ohshima, and R. M. Nerem. Application of the micropipette technique to the measurement of cultured porcine aortic endothelial cell viscoelastic properties. ASME J. Biomech. Eng. 112:263268, 1990.

${ }^{31}$ Seyedpour, S. M., M. Pachenari, M. Janmaleki, M. Alizadeh, and H. Hosseinkhani. Effects of an antimitotic drug on mechanical behaviours of the cytoskeleton in distinct grades of colon cancer cells. J. Biomech. 48:1172-1178, 2015.

${ }^{32}$ Sliogeryte, K., S. D. Thorpe, Z. Wang, C. L. Thompson, N. Gavara, and M. M. Knight. Differential effects of LifeAct-
GFP and actin-GFP on cell mechanics assessed using micropipette aspiration. J. Biomech. 49:310-317, 2016.

${ }^{33}$ Theret, D. P., M. J. Levesque, M. Sato, R. M. Nerem, and L. T. Wheeler. The application of a homogeneous halfspace model in the analysis of endothelial cell micropipette measurements. ASME J. Biomech. Eng. 110:190-199, 1988.

${ }^{34}$ Timoshenko, S. P., and J. N. Goodier. Theory of Elasticity (3rd edition). New York: McGraw-Hill Book Company, 1970.

${ }^{35}$ Vogel, V., and M. Sheetz. Local force and geometry sensing regulate cell functions. Nat. Rev. Mol. Cell Biol. 7:265-275, 2006.

${ }^{36}$ Wang, Z., A. K. T. Wann, C. L. Thompson, A. Hassen, W. Wang, and M. M. Knight. IFT88 influences chondrocyte actin organization and biomechanics. Osteoarthr. Cartil. 24:544-554, 2016.

${ }^{37}$ Xie, J. J., W. J. Hu, and J. L. Tao. Quasi-static experimental study on the energy dissipation performance of foam rubber based on quasi static. China Meas. Test. Technol. 38:29-32, 2012; ((In Chinese)).

${ }^{38}$ Zhang, Q. Y. Mechanical properties of chondrocytes from normal and osteoarthritic rabbit knee cartilage. Dissertation for the Master Degree of Taiyuan University of Technology, 2006 (In Chinese).

${ }^{39}$ Zhang, Q. Y., X. H. Wang, X. C. Wei, and W. Y. Chen. Characterization of viscoelastic properties of normal and osteoarthritic chondrocytes in experimental rabbit model. Osteoarthr. Cartil. 16:837-840, 2008.

${ }^{40}$ Zhou, E. H., C. T. Lim, and S. T. Quek. Finite element simulation of the micropipette aspiration of a living cell undergoing large viscoelastic deformation. Mech. Adv. Mater. Struct. 12:501-512, 2005. 